\title{
Erratum to: Modeling and analysis of passive dynamic bipedal walking with segmented feet and compliant joints
}

\author{
Yan Huang · Qi-Ning Wang · Yue Gao · Guang-Ming Xie
}

Published online: 2013

(C)The Chinese Society of Theoretical and Applied Mechanics and Springer-Verlag GmbH 2013

Erratum to: Acta Mechanica Sinica (2012) 28(5):1457-1465

DOI 10.1007/s10409-012-0079-6

In the electronic version of the original article, the is- sue number shown in the header of the article opening page was wrong. It should be Issue 5 as in Acta Mechanica Sinica (2012) 28(5):1457-1465.

The online version of the original article can be found at: http://dx.doi.org/10.1007/s10409-012-0079-6.

Y. Huang · Q.-N. Wang (凶) · Y. Gao · G.-M. Xie

Intelligent Control Laboratory, College of Engineering,

Peking University, 100871 Beijing, China

e-mail: qiningwang@pku.edu.cn 